\title{
Long noncoding RNA BFAL1 mediates enterotoxigenic Bacteroides fragilis-related carcinogenesis in colorectal cancer via the RHEB/ mTOR pathway
}

Yujie Bao ${ }^{1,2}$, Jiayin Tang $\mathbb{1}^{1,3}$, Yun Qian ${ }^{1}$, Tiantian Sun ${ }^{1}$, Huimin Chen ${ }^{1}$, Zhaofei Chen ${ }^{1}$, Danfeng Sun ${ }^{1}$, Ming Zhong ${ }^{3}$, Haoyan Chen', Jie Hong ${ }^{1}$, Yingxuan Chen (1) ${ }^{1}$ and Jing-Yuan Fang ${ }^{1}$

\begin{abstract}
Long noncoding RNAs (IncRNAs) contribute to many steps in carcinogenesis and often serve as biomarkers or therapeutic targets for tumor diagnosis and therapy. Although the role of IncRNAs in tumor formation is becoming clear, whether IncRNAs mediate gut microbiota-induced colorectal cancer (CRC) is largely unknown. Enterotoxigenic Bacteroides fragilis (ETBF) is a well-known tumor-inducing bacterium in the human gut; however, its tumorigenic effect remains to be explored. In the present study, we revealed the mechanism by which a IncRNA participates in gut bacteria-induced carcinogenesis: Bacteroides fragilis-associated InCRNA1 (BFAL1) in CRC tissues mediates ETBF carcinogenesis. BFAL1 was highly expressed in CRC tissues compared with that in adjacent normal tissues. In vitro, BFAL1 was upregulated in ETBF-treated CRC cells. Mechanistically, ETBF promoted tumor growth via BFAL1 by activating the Ras homolog, which is the MTORC1 binding/mammalian target of the rapamycin (RHEB/mTOR) pathway. Furthermore, BFAL1 regulated RHEB expression by competitively sponging microRNAs miR-155-5p and miR200a-3p. Clinically, both high expression of BFAL1 and high abundance of ETBF in CRC tissues predicted poor outcomes for patients with CRC. Thus, BFAL1 is a mediator of ETBF-induced carcinogenesis and may be a potential therapeutic target for ETBF-induced CRC.
\end{abstract}

\section{Introduction}

CRC is one of the most common malignant tumors worldwide, occurring in $5 \%$ of the adult population in the United States. Around 250,000 new cases are diagnosed each year, accounting for $\sim 9 \%$ of all malignancies in

\footnotetext{
Correspondence: Jing-Yuan Fang (jingyuanfang@sjtu.edu.cn)

${ }^{1}$ State Key Laboratory for Oncogenes and Related Genes; Key Laboratory of Gastroenterology \& Hepatology, Ministry of Health; Division of

Gastroenterology and Hepatology; Shanghai Institute of Digestive Disease; Renji Hospital, School of Medicine, Shanghai Jiao Tong University, 145 Middle Shandong Road, 200001 Shanghai, China

${ }^{2}$ Department of Infectious Disease, Shanghai Ninth People's Hospital, Shanghai Jiao Tong University School of Medicine, 639 Zhizhaoju Road, 200001 Shanghai, China

Full list of author information is available at the end of the article.

These authors contributed equally: Yujie Bao, Jiayin Tang

Edited by S. Inoue
}

Europe $^{1-4}$. CRC carcinogenesis is controlled by both genetic and environmental factors, in which the gut microbiota plays an important role in CRC formation ${ }^{5}$. Enterotoxigenic Bacteroides fragilis (ETBF) is one of the most prevalent species of carcinogenic bacteria in the colon $^{6}$. ETBF is a subtype strain of Bacteroides fragilis, which possesses $a$ bft gene, encoding Bacteroides Fragilis Toxin (BFT); the nontoxigenic Bacteroides fragilis (NTBF) subtype lacks the toxin gene ${ }^{7,8}$. Previous studies revealed that BFT targets the epithelial cell tight junctions, resulting in E-cadherin cleavage, enhanced barrier permeability, and Wnt/ $\beta$-catenin and nuclear factor kappa B (NF-kB) signaling9. A recent study showed that BFT promoted the normal-polyp-cancer process ${ }^{10}$. These mechanisms involved genetic mutations in various genes, such as ICAM1

\section{(c) The Author(s) 2019}

(c) (i) Open Access This article is licensed under a Creative Commons Attribution 4.0 International License, which permits use, sharing, adaptation, distribution and reproduction in any medium or format, as long as you give appropriate credit to the original author(s) and the source, provide a link to the Creative Commons license, and indicate if changes were made. The images or other third party material in this article are included in the article's Creative Commons license, unless indicated otherwise in a credit line to the material. If material is not included in the article's Creative Commons license and your intended use is not permitted by statutory regulation or exceeds the permitted use, you will need to obtain permission directly from the copyright holder. To view a copy of this license, visit http://creativecommons.org/licenses/by/4.0/. 
(intercellular adhesion molecule 1), $A R$ (androgen receptor), $J N K$ (JUN N-terminal kinase), MAPK (mitogen-activated protein kinase), and $N F-K B^{11-13}$.

Less than $2 \%$ of the human genome comprises proteincoding genes and the vast majority of transcripts consist of noncoding RNAs, representing a shift in our understanding of genome regulation that has emerged recently ${ }^{14}$. It is now apparent that the majority of cellular transcripts do not encode proteins, and many of these transcripts are long noncoding RNAs (lncRNAs) ${ }^{15}$. LncRNAs are transcribed mainly by RNA polymerase II, and are 5'-capped and polyadenylated like most RNAs, yet this class of transcripts has limited coding potential ${ }^{16}$. LncRNAs are involved in numerous biological functions and pathological processes, including development, proliferation, apoptosis, survival, differentiation, and carcinogenesis ${ }^{17,18}$, contributing to gene regulation by different mechanisms ${ }^{19}$. Among the reported mechanisms, some lncRNAs act post-transcriptionally as regulators of splicing, mRNA decay, protein translation, protein stability, or as molecular decoys for microRNAs ${ }^{20}$.

As omics-based technologies have matured, increasing evidence points to the microbial generation of bioactive compounds that affect the transcriptional machinery of host cells ${ }^{21}$. Recent studies have provided insights into the crosstalk between the gut microbiota and the host epigenome, including DNA methylation, histone modification, and noncoding $\mathrm{RNAs}^{22,23}$. Commensal microbiotaregulated lncRNAs have been identified in mouse models. Compared with germ-free mice, those that were colonized with specific bacteria displayed a significantly different lncRNA profile, with most of the lncRNAs being transcribed from introns. These lncRNAs contributed to the responses of intestinal epithelial cells to the bacteria ${ }^{24}$. However, these data were solely based upon the bioinformatic data. Therefore, we decided to identify an ETBFassociated lncRNA and explore its molecular mechanism in human CRC carcinogenesis to provide a potential therapeutic target for ETBF-induced CRC.

In the present study, Bacteroides fragilis-associated lncRNA1 (BFAL1) was identified. The expression profile of BFAL1 was validated and its function in ETBF-related carcinogenesis was investigated. BFAL1 mediates ETBFinduced tumor growth by activating the Ras homolog, which is the MTORC1 binding/mammalian target of the rapamycin (RHEB/mTOR) pathway. Further study showed that BFAL1 competitively bound to miRNAs miR155-5p and miR-200a-3p to upregulate RHEB expression. Clinicopathological information from 96 patients with CRC suggested that BFAL1 was an independent indicator of prognosis. Thus, the present study might identify a new field of research into how noncoding RNAs respond to microbial signaling and promote CRC carcinogenesis.

\section{Materials and methods \\ CRC tissue specimens}

The use of human tissues was performed in accordance with the Declaration of Helsinki and was approved by the ethics committee of Renji Hospital. Written informed consent was obtained from all participants in this study. Cohort 1 represented adult patients with CRC who underwent primary colorectal surgical resections at Renji Hospital and were enrolled from January 2010 to April 2014. All patients were diagnosed as colorectal adenocarcinomas. None of these patients had received radiotherapy or chemotherapy before surgery. Paired tissues (tumors and adjacent normal tissues) were collected and preserved in liquid nitrogen immediately for subsequent study.

\section{Detection of the amounts of ETBF in paired CRC tissues}

To detect the amounts of ETBF in CRC tissues, the total DNA was extracted from the paired CRC tissues by using a QIAamp DNA Mini Kit (QIAGEN, Hilden, Germany). DNA from each specimen was subjected to quantitative real-time PCR (qPCR) to detect the amounts of ETBF. The detected amount of the bft gene was normalized to that of the $16 \mathrm{~S}$ gene (Supplementary Material Table 1).

\section{Quantification of mRNAs and microRNAs}

The total RNA was isolated from cells by using the TRIzol reagent (Takara, Dalian, China) according to the manufacturer's protocol. Separation of the nuclear and cytoplasmic fractions was performed by using a PARIS ${ }^{\mathrm{m}}$ Kit (Invitrogen, Carlsbad, CA, USA). To obtain cDNA, $1 \mu \mathrm{g}$ of RNA was used as a template, and reverse transcription was performed by using a PrimeScript 1st strand cDNA Synthesis Kit (Takara) according to the manufacturer's instructions. Primers for LncRNAs and genes were designed and synthesized by Sangong Biotech, Shanghai, China (Supplementary Material Table 1). For miRNAs, $0.5 \mu \mathrm{g}$ of the total RNA was reverse transcribed into cDNA by using a specific miRNA stem loop primer. The levels of mRNA and miRNA were assessed quantitatively by using SYBR Green-based qPCR with specifically designed primers (GeneCopoeia, Rockville, MD, USA) (Supplementary Material Table 2). All qPCR reactions were performed by using a 7500 Fast Real-Time PCR System (Applied Biosystems), and all qPCR reagents were purchased from Takara. For each reaction, $1 \mu \mathrm{L}$ of the RT product was added to $10 \mu \mathrm{L}$ of $2 \times$ SYBGreen PCR Master Mix. Each sample was analyzed in triplicate. For lncRNAs and mRNAs, $A C T B$ (encoding beta actin) was used as an internal normalization control, and for the miRNAs, U6 was used as the internal normalization control. Relative quantification (RQ) was derived from the difference in the cycle threshold $(\mathrm{Ct})$ between the target 
RNA and internal controls $(\Delta \mathrm{Ct})$ as compared with control samples $(\Delta \Delta \mathrm{Ct})$ by using the equation $\mathrm{RQ}=2-\Delta \Delta \mathrm{Ct}$.

\section{Cell lines and cell culture}

Human CRC cell lines and the human normal colonic epithelial cell line FHC were purchased from American Type Culture Collection (ATCC, Rockefeller, MD, USA). Cells were cultured in a different medium according to the ATCC's indication (Gibco, Carlsbad, CA, USA). Cells were cultured in $5 \% \mathrm{CO}_{2}$ in a $37^{\circ} \mathrm{C}$ incubator.

\section{Bacteria strains and the growth condition}

The ETBF strain (ATCC 43860) and the NTBF strain ATCC $25285^{25,26}$ were purchased from ATCC. These two subtypes were cultured in the same medium and under anaerobic conditions. The anaerobic conditions were created by using a DG250 device (Don Whiteley Scientific, West Yorkshire, UK) and comprised an atmosphere of $90 \% \mathrm{~N}_{2}, 5 \% \mathrm{CO}_{2}$, and $5 \% \mathrm{H}_{2}$ at $37^{\circ} \mathrm{C}$. The anaerobic bacteria medium was prepared according to the ATCC indication (modified chopped meat medium). All the ETBF and NTBF treatment experiments in this article used the same bacterial concentration: a multiplicity of infection (MOI) of 500 .

\section{Overexpression of BFAL1 in CRC cells}

For ectopic expression, the full-length BFAL1 cDNA was subcloned into the vector pCDNA3.1 and transfected into HCT116 and DLD-1 cell lines by using the FuGENE HD transfection Reagent (Promega, Madison, WI, USA). The vectors were designed and constructed by Gene Pharma Company (Shanghai, China).

\section{Transfection of small-interfering RNAs and microRNA mimics and inhibitors}

The specified BFAL1 small-interfering RNAs (siRNAs) siRNA1 and siRNA2, and the specified RHEB small RNA, control siRNA were designed to knockdown BFAL1 or RHEB expression in cells. MiR-155-5p mimics and inhibitors and miR-200a-3p mimics and inhibitors were transfected to overexpress or knockdown the relevant microRNAs in cells. All these siRNA and miRNA mimics and inhibitors were designed and synthesized by Gene Pharma Company (Supplementary Material Table 3). Transfection was performed by using the DharmaFECT transfection reagent (GE, Boston, MA, USA) according to the manufacturer's instructions.

\section{Cell proliferation assays and the cell cycle test}

To assay cell proliferation, the CCK-8 (Cell Counting Kit-8, Dojindo, Japan) assay was used according to the manufacturer's instructions. Flow-cytometry analysis was conducted by using a BD LSR Fortessa instrument and PI/ RNase Staining Buffer (BD Biosciences, Lake Franklin, NJ,
USA) according to the manufacturer's instructions. The percentages of cells in different phases of the cell cycle were analyzed by using Flowjo software.

\section{Bioinformatic methods}

The potential microRNAs targeting RHEB were predicted and validated by using several online databases with different bioinformatic algorithms, such as TargetScan and RNAhybrid. We predicted the potential target genes of miR-155-5p and miR-200a-3p mainly based on a combination of $3^{\prime}$ UTR binding sites of target genes and the seed regions of miR-155-5p and miR-200a-3p. Furthermore, the minimum free energy (MFE) values of miRNA-lncRNA hybridization were calculated by using RNAhybrid software to evaluate the binding potential between BFAL1 and miR-155-5p or miR-200a-3p.

\section{High-throughput sequencing}

For RNA sequencing (RNA-seq), libraries were generated from the total RNA by using TruSeq RNA Sample Preparation v2, according to the manufacturer's protocol. Samples were sequenced on the Illumina HiScanSQ platform (Illumina, San Diego, CA, USA). Reads were mapped to the human genome (Hg19) by using TopHat v2.0.6 (Johns-Hopkins University, Baltimore, MD, USA), and mRNA quantification was performed by using Cuffdiff v2.0.2 (University of Maryland, College Park, MD, USA).

\section{Luciferase reporter assay}

To explore BFAL1's effect on the RHEB promoter transcriptional activity, a pGL3-RHEB reporter plasmid was designed and constructed (GENEray Company, Shanghai, China). The pGL3-RHEB plasmid and the relevant BFAL1 siRNAs or the pCDNA3.1 plasmid were transferred together into cells. To investigate the RHEB 3' UTR activity, three plasmids were designed and synthesized (GENEray): pmirGLO-RHEB 3' UTR wild-type (WT) plasmid, pmirGLO-RHEB 3' UTR miR-155-5p mut plasmid, and pmirGLO-RHEB 3' UTR miR-200a-3p mut plasmid. Luciferase activity was measured by using a FLUOstar device (Omega Engineering, Deckenpfronn, Germany), with the Dual-Luciferase reporter assay system (Promega).

\section{Western blotting and antibodies}

Proteins were extracted from cells after different treatments and quantified by using a BCA Protein Assay Kit (Thermo Fisher Scientific, West Palm Beach, FL, USA). Proteins $(40-60 \mu \mathrm{g})$ were electrophoresed through $10 \%$ SDS polyacrylamide gels and then electrophoretically transferred onto a polyvinylidene fluoride (PVDF) membrane (Millipore, Bedford, MA, USA). The primary antibodies included those recognizing RHEB (Abcam, 
Cambridge, UK and Cell Signaling Technology, Danvers, MA, USA), p70S6 Kinase (CST), and Phospho-p70S6 Kinase (CST); $\beta$-actin (CST) was used as an endogenous reference. All the secondary antibodies were labeled with horseradish peroxidase (HRP) (Aksomics, Shanghai, China). The signal was detected by using an ECL kit (Pierce Biotech, Rockford, IL, USA).

\section{ShRNA and adenovirus construction}

The shRNAs used in animal experiments and all adenoviruses were constructed and purchased from Obio Technology Company (Shanghai, China): Control shRNA (Y001, Obio), BFAL1-shRNA1 (Y2276, Obio), BFAL1shRNA2 (Y9601, Obio), BFAL1-overexpressing adenovirus (H8855, Obio), miR-155-5p adenovirus (H9491, Obio), and miR-200a-3p adenovirus (H9492, Obio).

\section{In vivo experiments}

All animal experiments were performed according to the National Institute of Health Guidelines for the Care and Use of Laboratory Animals. Our study was approved by the Animal Care and Use Committee of Renji Hospital, School of Medicine, Shanghai Jiao Tong University. Fiveweek-old male BALB/c nude mice were obtained from the Experimental Animal Center of Shanghai Institute for Biological Sciences (Shanghai, China). Each mouse was injected with $5.0 \times 10^{6}$ HCT116 cells subcutaneously at the right axilla to establish CRC xenograft tumors. Six days after inoculation, these mice were divided randomly into different groups for different treatments. Different treatments were delivered paratumorally at multiple points every 3 days. The tumor length and width were measured by using calipers every 3 days. The tumor volume was calculated and recorded using the volume formula (long dimension $\times$ wide dimension ${ }^{2} / 2$ ). After 21 days, all mice were killed and the subcutaneous xenograft tumors were excised and weighted. Finally, all tumors were kept in formalin for a further marker of proliferation Ki-67 (Ki-67) staining. The tumor volume and weight were presented as mean $\pm \mathrm{SD}(n=5$ or 6$)$.

\section{Statistical analysis}

Data were analyzed using Student's $t$-test for comparisons between groups to determine the statistical significance. The Pearson chi-squared test was used to analyze the associations between the patient's clinicopathological characteristics and ETBF abundance or BFAL1 expression. Kaplan-Meier analysis and the logrank test were performed to evaluate patient survival. A Cox proportional hazard model was performed to assess the prognostic value of ETBF and BFAL1. The difference between the growth rates was determined using analysis of variance (ANOVA) with repeated measures analysis of variances. All statistical tests were performed using SPSS
20.0 statistical software (IBM Corp., Armonk, NY, USA) or Graphpad Prism 5.0 (Graphpad Software Inc., San Diego, CA, USA). Each experiment was repeated at least three times. All data were presented as the mean \pm SD and were calculated from three separate experiments. The results were considered statistically significant when the two-tailed $P$ value was $<0.05$.

\section{Results}

BFAL1 is highly expressed in ETBF-related CRC tissues and cells

To identify a specific IncRNA associated with ETBFrelated CRC, the top 20 significant CRC-related lncRNAs (tumors vs. normal, false discovery rate $(\mathrm{FDR})<0.01$, fold change $>1.5$ ) were targeted in the GEO database GSE20916. After that, these 20 lncRNAs were further filtrated in another GEO database GSE31737 (tumors vs. normal, FDR $<0.01$ ) and finally we got the eight lncRNAs (Fig. S1A). To search for lncRNAs associated with ETBF among these eight candidates, two colorectal cancer cell lines, DLD-1 and HCT116, were treated with ETBF for up to $30 \mathrm{~h}$. Interestingly, significantly increased expressions of lncRNAs AK096729 (BFAL1), AK001058, and AK098081 were detected in the ETBF-treated HCT116 and DLD-1 cells after $24 \mathrm{~h}$ (Fig. 1a, Fig. S1B), compared with those in NTBF or simple medium-treated cells. This phenomenon indicated that ETBF increases the expression of certain lncRNAs in CRC cells.

To identify the expression profile of these three ETBFassociated lncRNAs in CRC tissues, 96 cases (Renji Cohort 1) were adopted to test both the ETBF abundance and lncRNA expression levels in the cancer tissues paired with noncancerous tissues. The data showed that cancer tissues presented significantly higher expression levels of AK096729 ( $P=0.0123$, Fig. 1b), AK001058 $(P=0.0418)$, and AK098081 $(P=0.0289)$ (Fig. S1C) and simultaneously had a higher abundance of ETBF $(P=0.0004$, Fig. 1c) compared with those in normal tissues. Furthermore, the correlation between the ETBF abundance and the expression of these IncRNAs in cancer tissues was detected. The results showed that cancer tissues with a relatively high ETBF colonization presented a higher enrichment of AK096729 ( $P=0.01$, Fig. 1d), whereas AK001058 and AK098081 levels were not obviously related with ETBF abundance (Fig. S1D). Therefore, we focused on AK096729 for further study and named it as Bacteroides fragilis-associated lncRNA1 (BFAL1).

In order to identify whether BFAL1 is a novel lncRNA, the full-length cDNA of BFAL1 was isolated by using $3^{\prime}$ and $5^{\prime}$ rapid amplification of cDNA ends (RACE) and sequenced (Fig. S1E). BFAL1 is located on chromosome 19 and is mainly distributed in the cell cytoplasm (Fig. S1F). To determine its noncoding character, the BFAL1 sequence was analyzed by using an ab initio ncRNA 

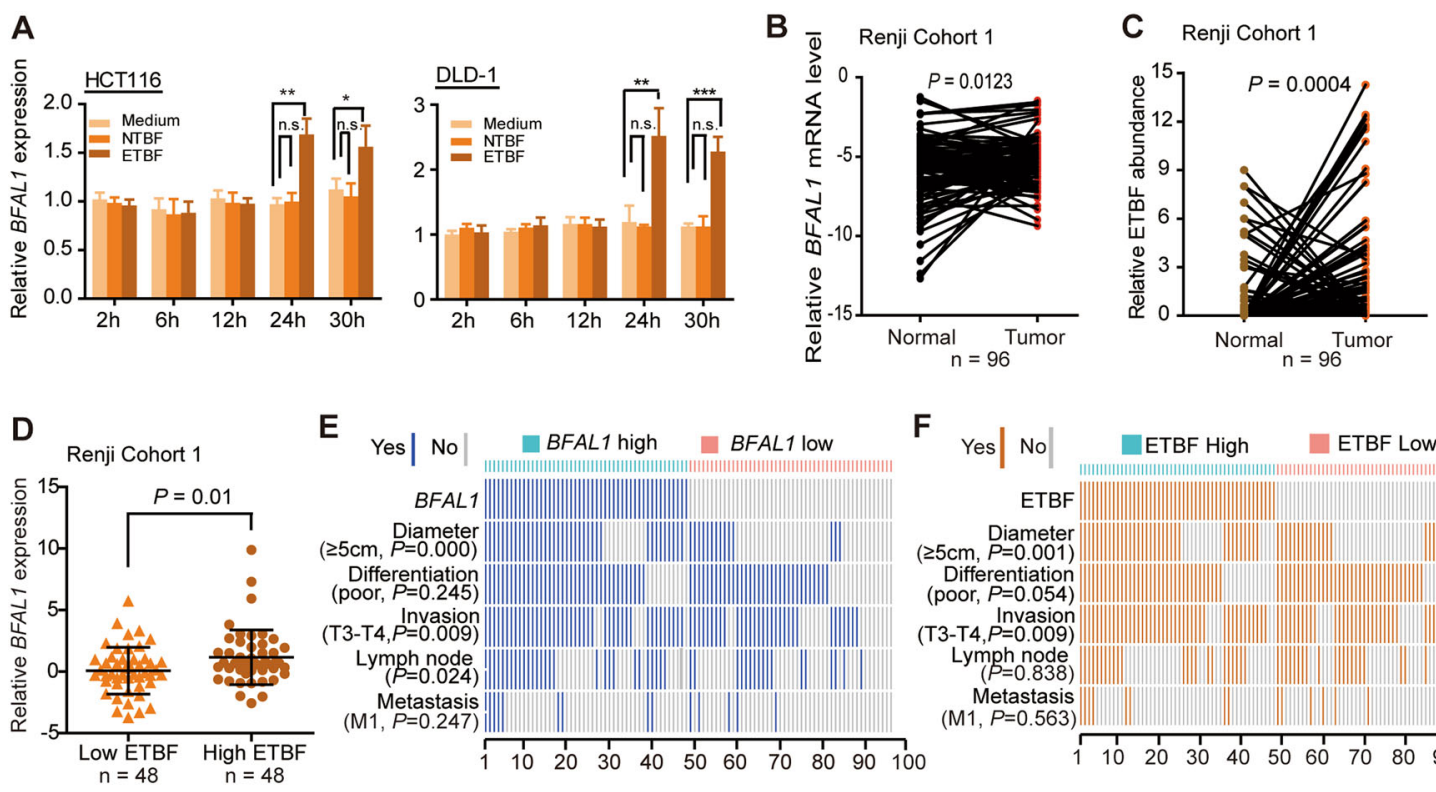

E

Yes $\mid$ No $\quad$ BFAL1 high $\quad B F A L 1$ low

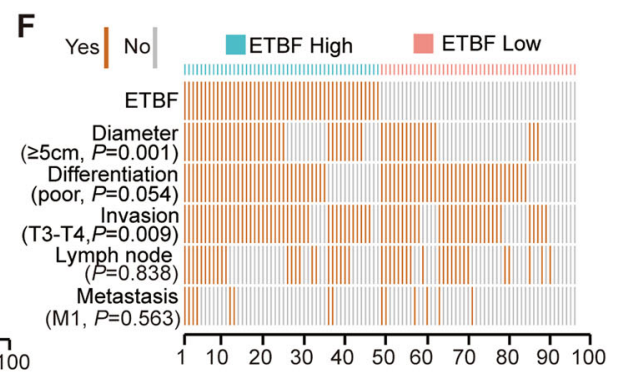

G

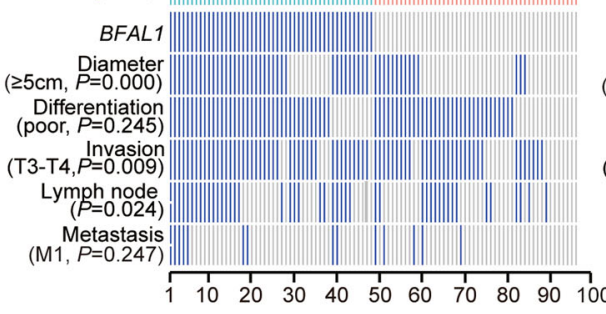

Genji Cohort 1

H Renji Cohort 1

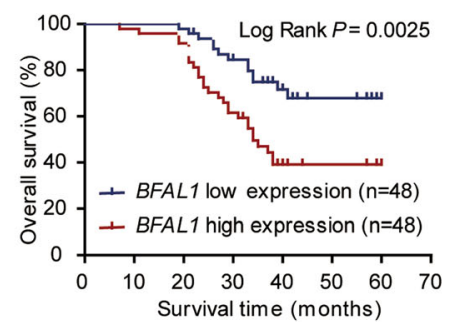

I

Renji Cohort 1

Multivariate risk factors

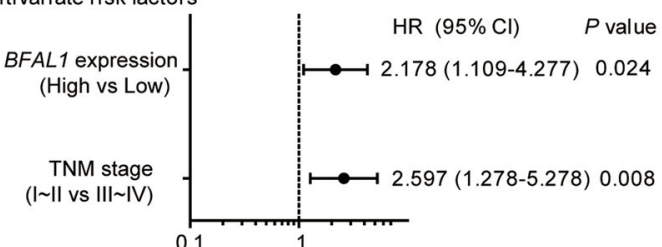

K Renji Cohort 1

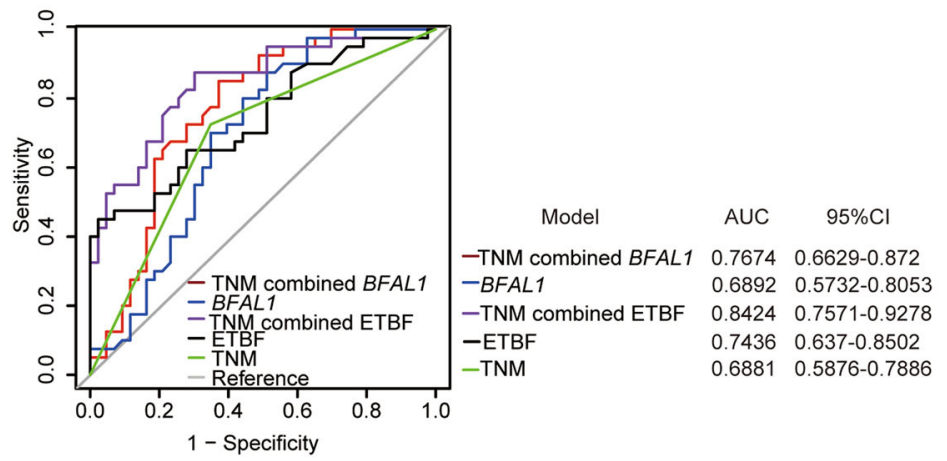

J

Renji Cohort 1

Multivariate risk factors

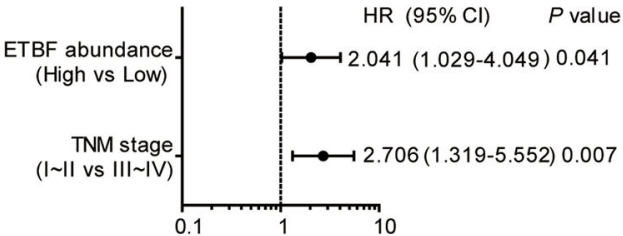

Fig. 1 (See legend on next page.) 
(see figure on previous page)

Fig. 1 BFAL1 is upregulated by ETBF and both of them are clinicopathologically related to CRC features and outcomes. a The mRNA level of BFAL1 in ETBF or NTBF-treated HCT116 cells and DLD-1 cells at different time points, compared with cells in a single bacterial medium (mean \pm SD of three independent experiments; Student's $t$-test, ${ }^{*} P<0.05$, $\left.{ }^{*} P<0.01,{ }^{* * *} P<0.001\right)$. b Comparison of BFAL1 mRNA levels in CRC tumor tissues and pair-matched normal tissues in Renji Cohort $1(n=96$, Student's $t$-test, $P<0.05)$. c Relative DNA abundance of ETBF in tumor tissues and pairmatched normal tissues, Renji Cohort $1(n=96$, Student's $t$-test, $P<0.001)$. d Comparison of BFAL1 mRNA levels between high ETBF abundance tissues $(n=48)$ and low ETBF abundance tissues $(n=48)$ (Student's $t$-test, $P<0.05)$. e Comparing the tumor diameter, pathological differentiation, invasion depth, lymph node involvement, and vascular metastasis between BFAL1 high and low tumors in Renji Cohort 1. The association of different clinicopathological features was illustrated in a heatmap (statistical significance was performed using the $x^{2}$ test). $\mathbf{f}$ The correlation of different clinicopathological features with ETBF high- and low-abundance tumors $\left(X^{2}\right.$ test). gOverall survival of patients with CRC patients with high or low BFAL1 expression in Renji cohort 1, Kaplan-Meier survival analysis ( $P=0.0025$; HR 2.656; 95\% Cl: 1.409-5.007). h Overall survival of patients with CRC with high or low ETBF abundance in Renji Cohort 1, Kaplan-Meier survival analysis ( $P=0.007$; HR 2.351; $95 \%$ Cl: $1.281-4.462)$. i, j Multivariate regression analysis of Renji Cohort 1. i included all the CRC clinicopathological factors. $\mathbf{j}$ Excluded the factor of BFAL 1 expression. $\mathbf{k}$ ROC analysis based on the ETBF abundance, BFAL1 expression, and TNM stage in Renji Cohort 1 (bars correspond to 95\% confidence intervals)

transcriptome predictor, which showed that its noncoding probability was $95.22 \%$, whereas its coding probability was $4.78 \%$ (Fig. S1G). Analysis of BFAL1 ORF sequences using a coding potential calculator failed to find any protein-coding potential (Fig. S1H). In addition, we calculated its coding potential using PhyloCSF. The PhyloCSF score of BFAL1 was -774 , indicating that BFAL1 is unlikely to encode any protein product. Analysis using qPCR showed a higher expression of BFAL1 in human CRC cells than in normal colorectal epithelial cells (FHC) (Fig. S1I).

\section{Both ETBF and BFAL1 are associated with patients' clinicopathology and outcomes}

To analyze the relationship between BFAL1 and the clinicopathological features of CRC, correlation regression analysis was used. Detailed information regarding Renji Cohort 1 is shown in Table S1. The data showed that the expression of BFAL1 was positively related with tumor size $(P=0.000)$, tumor invasion $(P=0.009)$, and lymph node involvement $(P=0.024)$ (Fig. 1e). Similarly, ETBF abundance also showed a positive relationship with tumor size $(P=0.001)$ and invasion $(P=0.009)$ (Fig. 1f). We further examined the outcomes of these 96 cases. Kaplan-Meier analysis revealed that high expression of BFAL1 in tumor tissues was associated with a reduced overall survival ( $P=0.0025$; hazard ratio (HR) 2.656; $95 \%$ confidence interval (CI) 1.409-5.007) (Fig. 1g). Patients with high ETBF abundance in their tumor tissues also exhibited a poor outcome ( $P=0.007$; HR 2.351 ; $95 \%$ CI 1.281-4.462) (Fig. 1h). Univariate regression analysis showed that both ETBF abundance $(P=0.002$; HR 2.358; 95\% CI 1.230-4.522) and BFAL1 expression $(P=0.004$; HR 2.642; 95\% CI 1.361-5.129) had the potential to predict CRC prognosis (Fig. S1J). Further multivariate regression analysis showed that BFAL1 expression $(P=$ 0.024; HR 2.178; 95\% CI 1.109-4.277) and ETBF abundance $(P=0.041$; HR $2.041 ; 95 \%$ CI $1.029-4.049)$ were two independent factors for CRC aggressiveness, with significant hazard ratios for predicting outcomes (Fig. 1i, j).
Receiver-operating characteristic (ROC) analysis illustrated that the area under curve (AUC) of the TNM-stage-based model combined with BFAL1-based prediction (0.7674) was higher than that of the single TNM-stage model (0.6881), as was the combination of the TNM stage and ETBF prediction (0.8424) (Fig. 1k). This suggested that the combination of BFAL1 or ETBF and TNM stage was more accurate to predict CRC prognosis than the TNM stage alone.

\section{BFAL1 mediates ETBF's promotion of tumor growth in vitro and in vivo}

To evaluate the biological function of BFAL1 in CRC, we knocked down BFAL1 using BFAL1-siRNA1 in CRC cells and extracted RNA for RNA-seq analysis. A total of 14,737 downregulated genes and 15,913 upregulated genes ( $\geq 2$-fold) were detected after the knockdown of BFAL1 in CRC cells (raw data are accessible via the GEO number: GSE129950). Gene set enrichment analysis (GSEA) revealed that the genes related to cell proliferation were reduced (normalized enrichment score (NES) = $1.80, P<0.01$; Fig. S2A) and cell cycle-associated pathways were downregulated $(\mathrm{NES}=2.80, P<0.01$; Fig. $\mathrm{S} 2 \mathrm{~B}$ ) in BFAL1 knockdown cells. Meanwhile, we also treated DLD-1 cells with ETBF for $24 \mathrm{~h}$ and extracted the total human RNA for RNA-seq analysis. We identified 350 upregulated and 154 downregulated genes ( $\geq 2$-fold and FDR $<0.05$; raw data accessible via the GEO number: GSE130152). Gene ontology (GO) enrichment analysis also revealed that ETBF treatment regulated a set of genes associated with cell proliferation and the cell cycle (Fig. S2C). These two RNA-seq analyses suggested that both $B F A L 1$ and ETBF have an effect on CRC cell growth.

To validate the biological function of BFAL1 and ETBF in vitro, CCK-8 assays were performed in HCT116 and DLD-1 cells. The results showed that ETBF enhanced cell proliferation, whereas NTBF and single-medium treatment had no effect on proliferation (Fig. 2a). Meanwhile, we tested the function of $B F A L 1$ by overexpression from a $B F A L 1$ plasmid (Fig. S2D) or knockdown with BFAL1siRNA1/2 (Fig. S2E). Overexpression of BFAL1 promoted 

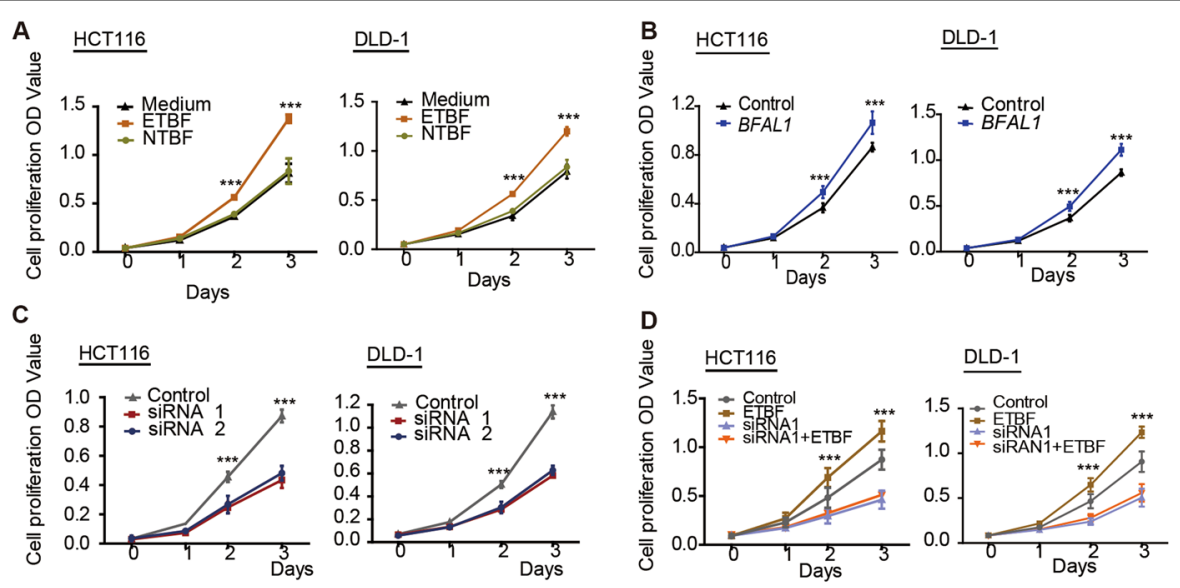

E $\quad \underline{\text { HCT116 }}$
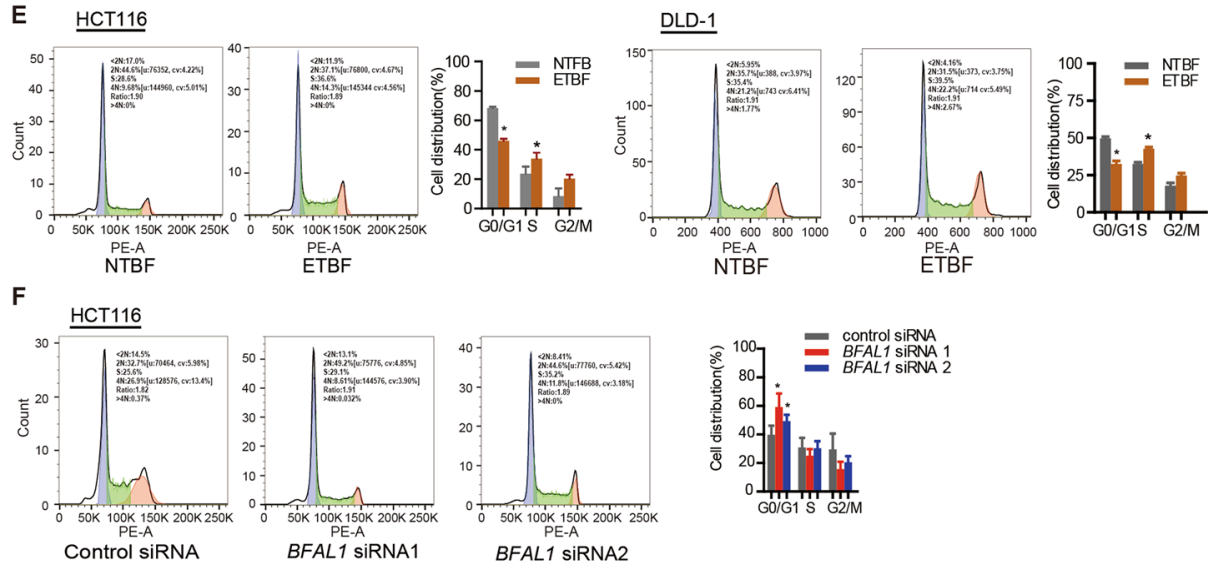

$$
\underline{\text { DLD-1 }}
$$
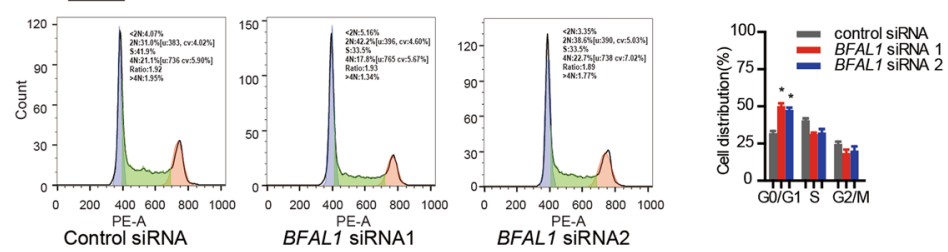

G
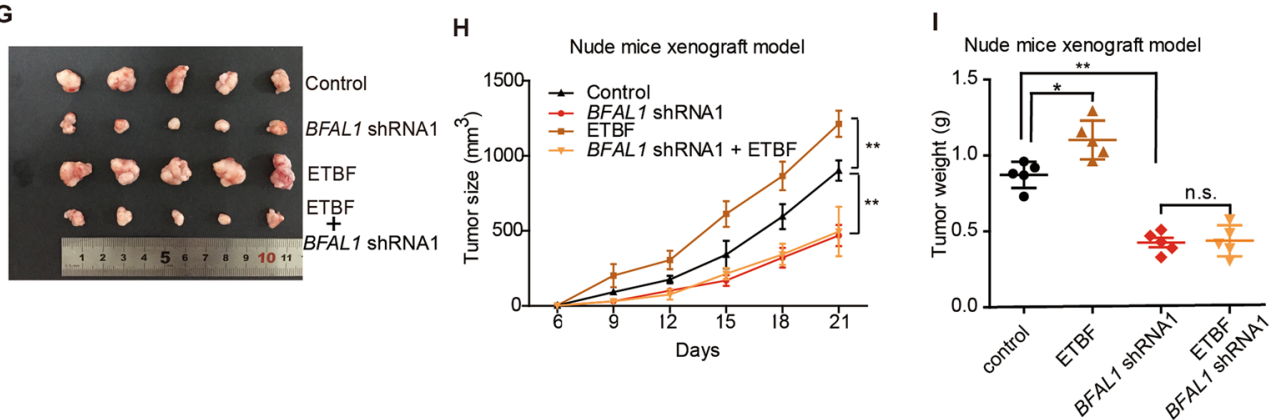

Fig. 2 ETBF exerts a biological function on CRC cell growth via BFAL1 in vitro and in vivo. a CCK-8 assay of ETBF-treated HCT116 cells and DLD1 cells compared with NTBF or single bacterial medium-treated cells $\left(n=6\right.$, ANOVA, $\left.{ }^{* *} P<0.001\right)$. b CCK-8 assay of BFAL 1 overexpression and control cells $\left(n=6\right.$, ANOVA, $\left.{ }^{* * *} P<0.001\right)$. c CCK-8 assay of BFAL 1 knockdown in HCT116 cells and DLD-1 cells $\left(n=6\right.$, ANOVA, $\left.{ }^{* * *} P<0.001\right)$. $\mathbf{d}$ CCK-8 assays of ETBF-treated, BFAL1 knockdown HCT116 cells and DLD-1 cells $\left(n=6\right.$, ANOVA, $\left.{ }^{* * *} P<0.001\right)$. e Cell cycle analysis of ETBF-treated HCT116 cells and DLD-1 cells (mean \pm SD of three independent experiments; ANOVA, $\left.{ }^{*} P<0.05\right)$. f Cell cycle analysis of BFAL1 knockdown of HCT116 cells and DLD-1 cells (mean \pm SD of three independent experiments; ANOVA, ${ }^{*} P<0.05$ ). $\mathbf{g}$ Xenograft tumors in the nude mouse model under different treatments $(n=5)$. h Statistical analysis of tumor sizes (mean $\pm S D, n=5$, ANOVA, $\left.{ }^{* *} P<0.01\right)$. i Tumor weights of different mouse groups (mean $\pm S D, n=5$, ANOVA, $\left.{ }^{*} P<0.05,{ }^{* *} P<0.01\right)$ 
cell proliferation (Fig. 2b), whereas BFAL1 knockdown suppressed cell proliferation (Fig. 2c). Interestingly, ETBFinduced proliferation was significantly suppressed by BFAL1 knockdown (Fig. 2d), indicating that the ability of ETBF to promote cell proliferation might depend on $B F A L 1$. Flow- cytometry assays were performed to test the cell cycle process affected by ETBF and BFAL1. The results showed that cell growth was accelerated by ETBF compared with treatment with NTBF (Fig. 2E). In addition, knockdown of BFAL1 blocked the cell cycle (Fig. 2f).

In vivo, a xenograft tumor model was established in BALB/c nude mice. We observed that ETBF-treated tumors were larger and heavier than those of the control, whereas BFAL1-shRNA1/2 virus-treated tumors were smaller and lighter. However, ETBF treatment could not rescue the tumor growth inhibited by BFAL1shRNA1/2 virus treatment (Fig. 2g-i, Fig. S2G-I). Ki-67 staining was consistent with these observations (Fig. S2F, $\mathrm{J})$. From these in vitro and in vivo experiments, we concluded that ETBF exerts its effects on CRC tumor growth through BFAL1.

\section{BFAL1 mediates ETBF-induced tumor growth by activating the RHEB/mTOR pathway}

To further explore the mechanism of BFAL1 in ETBFrelated CRC cell growth, we reviewed the two RNA-Seq data sets mentioned above. Both Hallmark and Kyoto Encyclopedia of Genes and Genomes (KEGG) enrichment analysis in GSEA showed that knockdown of BFAL1 affected mTOR-associated gene sets (Fig. 3a). KEGG analysis of ETBF-treated cell data also demonstrated that the mTOR-signaling pathway is the significantly functional pathway (Fig. 3b). Taken together, the results suggested that the mTOR signaling is the common downstream pathway of both ETBF and BFAL1. We then explored how ETBF and BFAL1 activate the mTOR pathway. According to the KEGG analysis, the mTORsignaling pathway in GSEA comprised 12 associated genes affected by BFAL1 knockdown: VEGFB, EIF4EBP1, PGF, CAB39L, MAPK1, FIGF, ULK2, PIK3CD, AKT3, EIF4E, MLST8, and RHEB (Table S2). To screen out the ETBF and BFAL1-targeted gene, we treated DLD-1 cells with ETBF for $24 \mathrm{~h}$ and extracted the cell's RNA for gene expression analysis using qPCR. Among the 12 genes, only the RHEB mRNA level was upregulated by ETBF (Fig. 3c); the others showed no significant response to ETBF (Fig. S3A). Therefore, RHEB became our research focus. RHEB can bind directly to the mTOR complex and regulate the mTOR-signaling pathway by phosphorylating the p70S6 Kinase (S6K); ${ }^{27}$ therefore, we hypothesized that ETBF and BFAL1 might target RHEB to regulate the mTOR pathway. First, we validated the qPCR result from the ETBF-treated cells by showing that overexpression of $B F A L 1$ upregulated the RHEB mRNA level (Fig. 3d), whereas knockdown of BFAL1 downregulated its level (Fig. 3e). We then confirmed these findings using western blotting. The results demonstrated that the expression levels of RHEB and Phospho-S6k, but not the total S6K, were increased in ETBF-treated cells compared with those in NTBF-treated cells (Fig. 3f). Similar results were obtained in BFAL1-overexpressing cells (Fig. 3g). Conversely, the expression levels of RHEB and Phospho-S6k were decreased in BFAL1-knockdown cells (Fig. 3h). Furthermore, ETBF could not upregulate RHEB and Phospho-S6K levels after knockdown of BFAL1 in DLD-1 cells (Fig. 3i). Also, BFAL1 was not likely to upregulate P-S6K expression after $R H E B$ knockdown (Fig. 3j).

\section{$B F A L 1$ regulates $R H E B$ expression by binding to miR-155-5p and miR-200a-3p}

We next explored the mechanism of how BFAL1 regulates the $R H E B$ expression. The RHEB mRNA level was regulated by $B F A L 1$ expression; therefore, we synthesized a recombinant luciferase reporter containing the $R H E B$ promoter region to test the promoter activity. However, $B F A L 1$ had no effect on the promoter activity of $R H E B$ (Fig. S3B), suggesting that BFAL1 might regulate RHEB mRNA expression via a post-transcriptional mechanism. Recently, it has been reported that miRNAs direct sequence-specific cleavage of the target mRNA and repress its translation, and many RNA transcripts block this activity by sponging miRNA, permitting target mRNA translation as competing endogenous RNAs (ceRNA) ${ }^{28,29}$. The miRNAs that might target RHEB were predicted by the TargetScan prediction algorithm ${ }^{30}$ (http://www. targetscan.org) and then were validated in RNAhybrid ${ }^{31}$ (http://bibiserv.techfak.uni-bielefeld.de/rnahybrid) to calculate the complete hybridization around the seed match with the RHEB 3' UTR. Finally, miR-155-5p and miR200a-3p were screened out, targeting the RHEB 3' UTR and also showed high MFE values of hybridization with $B F A L 1$ (Fig. 4a). Several studies have reported that miR$155-5 p$ and miR-200a-3p target RHEB mRNA ${ }^{32-36}$. In the present study, mimics and inhibitory forms of miR-155-5p and miR-200a-3p were synthesized and transfected into CRC cells. Inhibitors of miR-155-5p and miR-200a-3p increased both the mRNA (Fig. 4b) and protein levels (Fig. 4c) of RHEB, whereas their mimics decreased RHEB mRNA and protein levels (Fig. 4d, e). To further test the miRNA effect on the 3' UTR of RHEB, pmirGLO-RHEB wild-type plasmids and inhibitors of miR-155-5p and miR-200a-3p were co-transfected into CRC cells. The results showed that the RHEB 3' UTR activity was significantly enhanced by the miRNA inhibitors, not the mutated one (Fig. $4 \mathrm{f}-\mathrm{h}$ ). By contrast, mimics of miR-155$5 \mathrm{p}$ and miR-200a-3p exerted the opposite effect on RHEB $3^{\prime}$ UTR activity reporter (Fig. 4i and j). Thus, we 


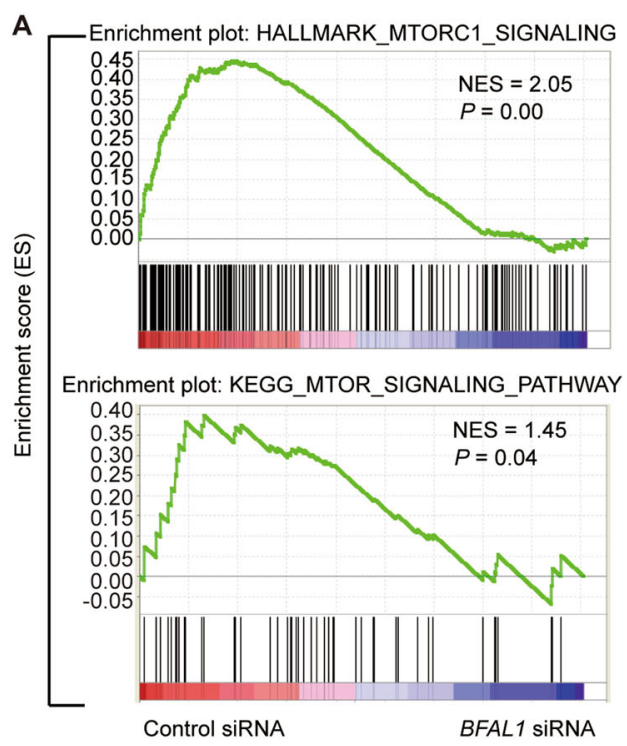

B

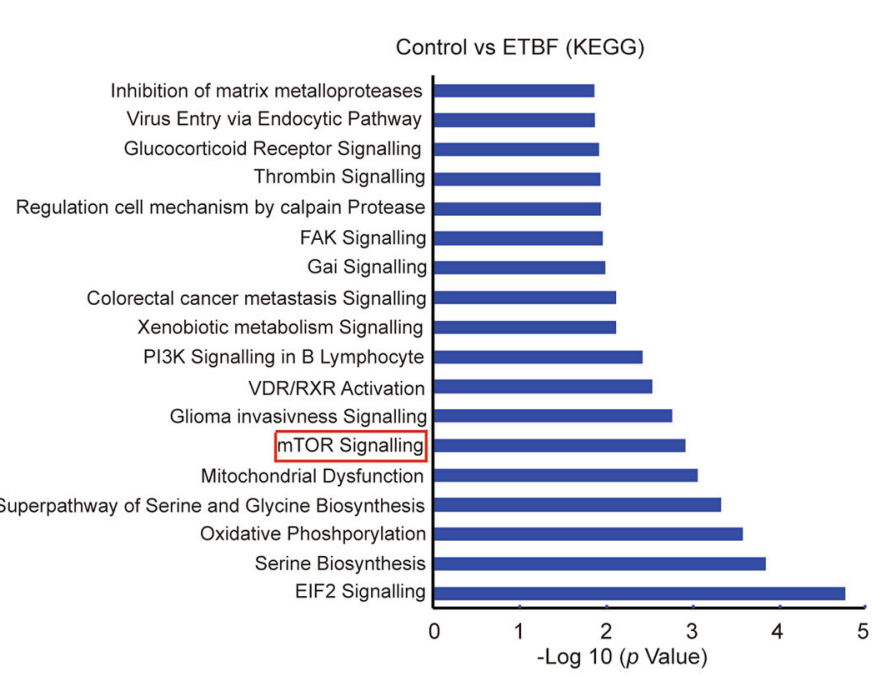

C
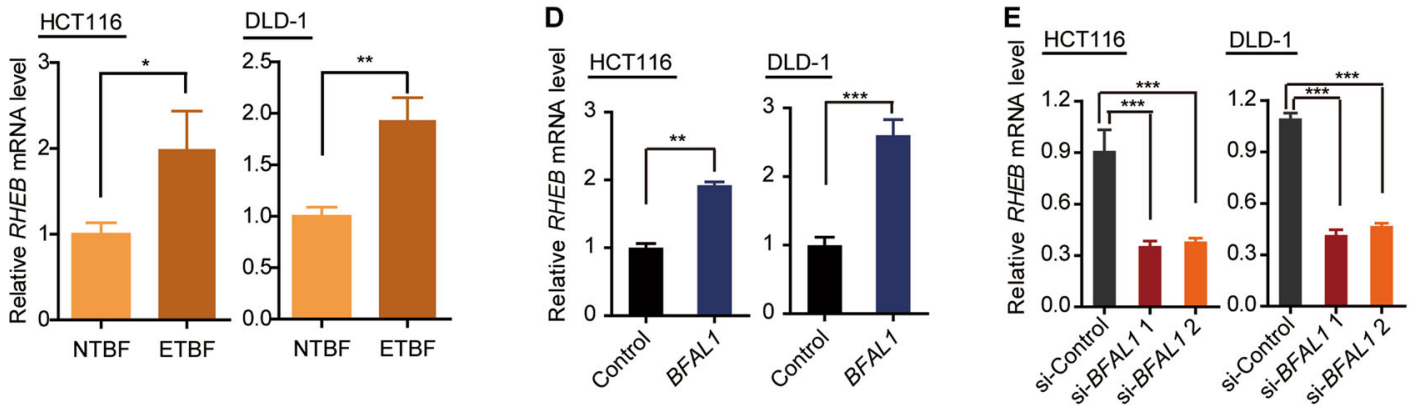

$\mathbf{F}$
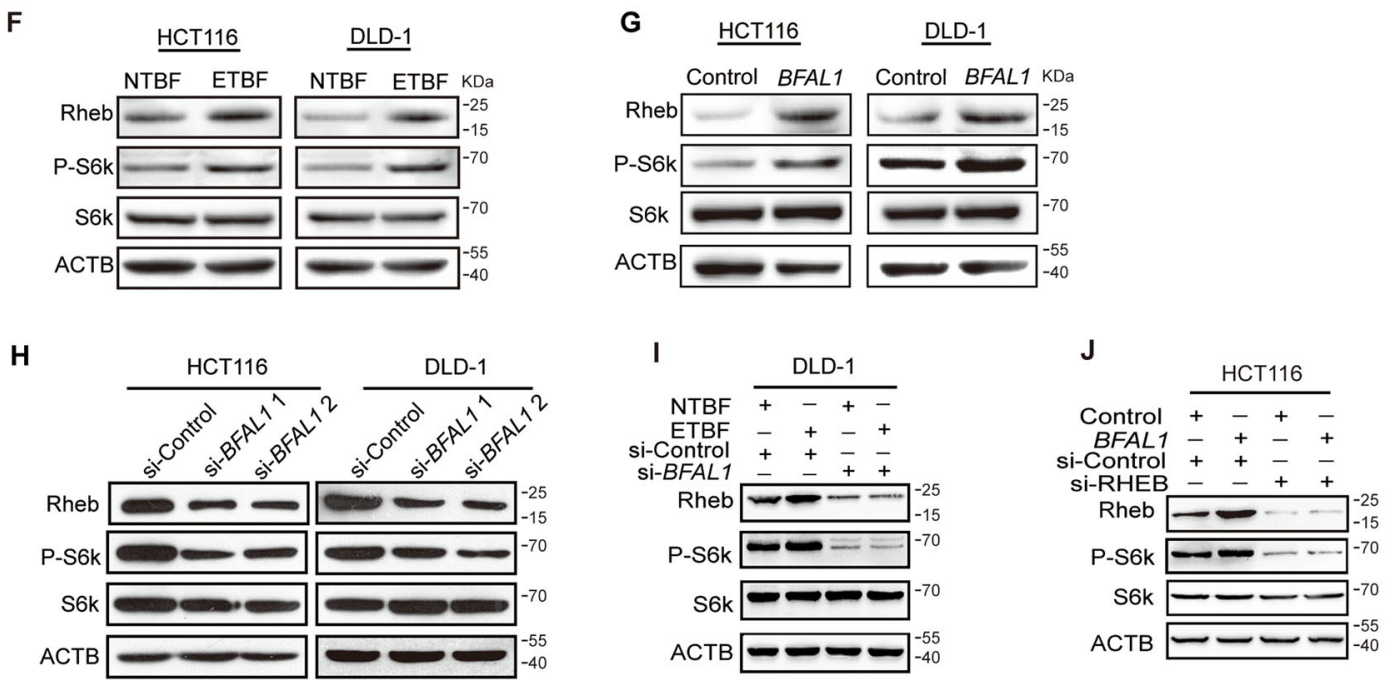

Fig. 3 ETBF activates the RHEB/mTOR-signaling pathway via BFAL1 in CRC. a GSEA analysis: enrichment hallmark of mTORC1 signaling (NES = 2.05, $P=0.00$ ) and KEGG mTOR-signaling pathway (NES $=1.45, P<0.05)$. $\mathbf{b}$ KEGG pathway analysis of ETBF-treated DLD-1 cells. (c) The mRNA level of RHEB in ETBF-treated HCT116 cells and DLD-1 cells compared with that in NTBF-treated cells. d The RHEB mRNA level in BFAL1-overexpressing HCT116 cells and DLD-1 cells. e The RHEB mRNA level in BFAL1-knockdown HCT116 cells and DLD-1 cells. $\mathbf{f}$ The protein expression of the RHEB/mTOR pathway in ETBF-treated HCT116 cells and DLD-1 cells, compared with that in NTBF-treated cells. $\mathbf{g}$ The expression of the RHEB/mTOR pathway in BFAL1-overexpressing HCT116 cells and DLD-1 cells. $\mathbf{h}$ The expression of the RHEB/mTOR pathway in BFAL 1-knockdown HCT116 cells and DLD-1 cells. $\mathbf{i}$ The expression of the RHEB/mTOR pathway in DLD-1 cells treated with ETBF after BFAL 1 knockdown. $\mathbf{j}$ The expression of the RHEB/mTOR pathway in HCT116 cells overexpressing BFAL1 after RHEB knockdown 


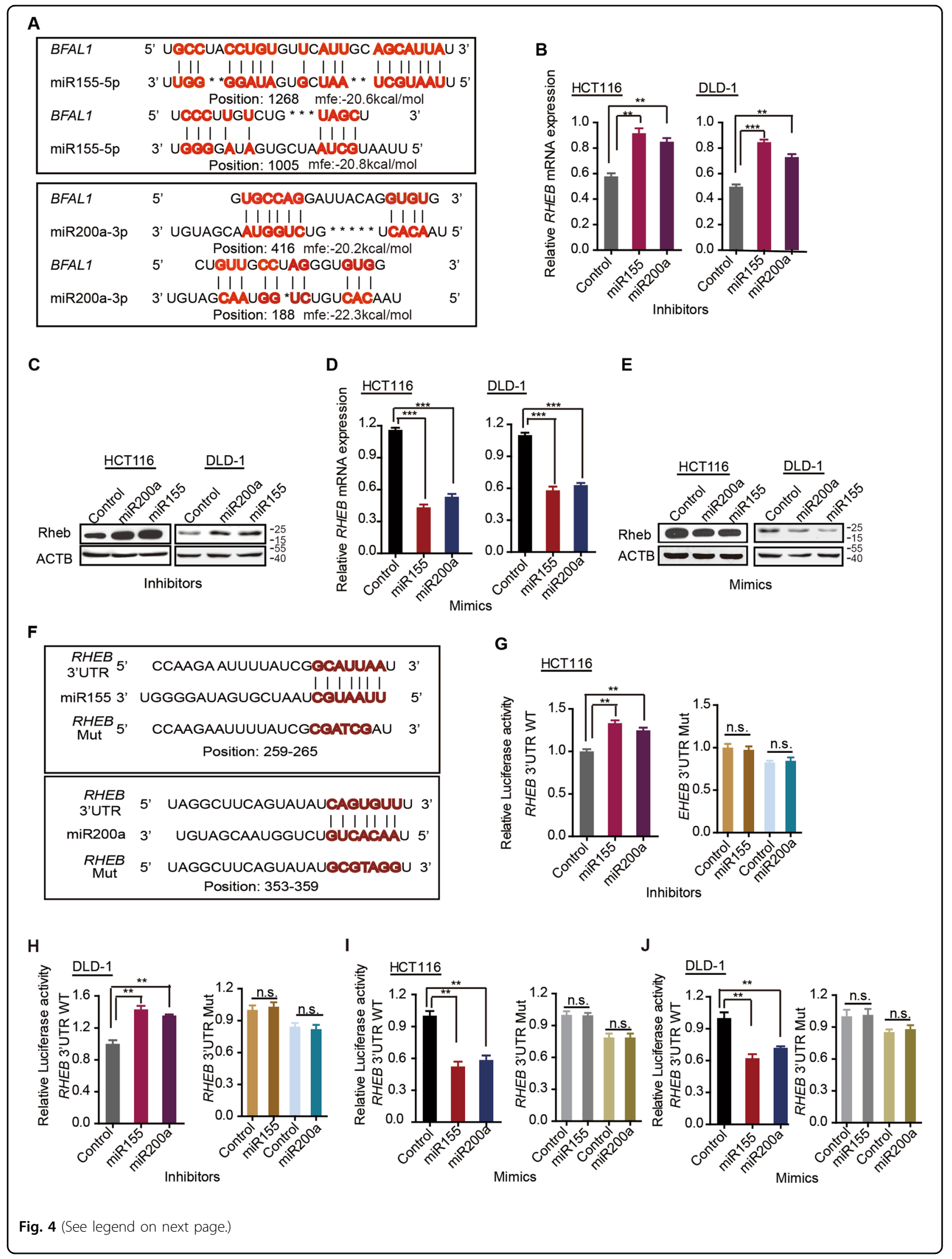


(see figure on previous page)

Fig. 4 miR-155-5p and miR-200a-3p target the RHEB 3' UTR. a The predicted binding sites of miR-155-5p and miR-200a-3p on the BFAL 1 transcripts. b RHEB mRNA levels in HCT116 cells and DLD-1 cells treated with inhibitors of miR-155-5p or miR-200a-3p. c RHEB levels in HCT116 cells and DLD-1 cells transfected with inhibitors of miR-155-5p or miR-200a-3p. d RHEB mRNA levels in HCT116 cells and DLD-1 cells transfected with mimics of miR-155-5p or miR-200a-3p. e RHEB levels in HCT116 cells and DLD-1 cells transfected with mimics of miR-155-5p or miR-200a-3p. $\mathbf{f}$ The predicted miR-155-5p and miR-200a-3p binding sites on the RHEB $3^{\prime}$ UTR and the mutated sites. $\mathbf{g}, \mathbf{h}$ Luciferase reporter assays were performed in HCT116 cells and DLD-1 cells transfected with inhibitors of miR-155-5p or miR-200a-3p. The luciferase reporters expressing wild-type or mutant human RHEB 3' UTR were used (data represent mean \pm SD of three independent experiments, ANOVA, ${ }^{* *} P<0.01$ ). $\mathbf{i}, \mathbf{j}$ Luciferase reporter assays were performed in HCT116 cells and DLD-1 cells transfected with mimics of miR-155-5p or miR-200a-3p. The luciferase reporters expressing wild-type or mutant human RHEB 3' UTR were used (data represent mean \pm SD of three independent experiments, ANOVA, ${ }^{* *} P<0.01$ )

confirmed that both miR-155-5p and miR-200a-3p target the 3' UTR of RHEB to repress its expression.

Next, we investigated whether BFAL1 regulated the RHEB expression via miR-155-5p and miR-200a-3p. qPCR analysis showed that overexpression of BFAL1 decreased miR-155-5p and miR-200a-3p mRNA levels (Fig. 5a), whereas knockdown of BFAL1 increased their levels (Fig. 5b). Furthermore, luciferase reporter assays revealed that overexpression of $B F A L 1$ led to activation of the RHEB 3' UTR (Fig. 5c), whereas knockdown of BFAL1 led to deactivation of the RHEB 3' UTR (Fig. 5d). Collectively, the results strongly suggested that BFAL1 sponged miR-155-5p and miR-200a-3p to regulate $R H E B$ expression acting as a ceRNA ${ }^{37,38}$.

In vivo, we also confirmed that miR-155-5p and miR200a-3p had biological functions using a xenograft tumor model. The results showed that transfection with viruses expressing miR-155-5p and miR-200a-3p obviously attenuated tumor growth compared with the controls. Although BFAL1-overexpressing virus and ETBF treatment enhanced tumor growth, they could not entirely recover the growth retardation induced by the miR-155$5 p$ and miR-200a-3p mimics (Fig. 5e-g, Fig. S3C). Collectively, these results confirmed that BFAL1 binds competitively with miR-155-5p and miR-200a-3p to attenuate their suppressive function on $R H E B$ expression.

In conclusion, we highlighted a new signaling cascade of ETBF-BFAL1-RHEB/mTOR that promotes tumor growth in CRC (Fig. 6).

\section{Discussion}

The gut microbiota, as a complicated symbiotic organ in mammals, has marked effects on host health and disease. The gut microbiome provides environmental cues to which the host responds via alterations in the host epigenome and gene expression ${ }^{22}$. Previous studies have focused on the roles of protein-coding genes. However, increasing evidence has demonstrated a crosstalk between the microbiota and epigenetic alterations ${ }^{39}$. DNA methylation, histone modifications, and various noncoding RNAs are involved in bacteria-related cancers ${ }^{40,41}$. However, the regulatory mechanism remains largely unknown. ETBF is regarded as one of the most prominent bacterial species in human CRC. Although it has been studied for many years, the mechanism by which ETBF alters the host's noncoding RNAs remains poorly understood. Through a series of genomic, bioinformatic, biological, xenograt model, and clinical studies, we identified that lncRNA BFAL1 is upregulated by ETBF and revealed a new mechanism by which BFAL1 participates in ETBFinduced CRC formation.

A study from Turkey has examined ETBF in a CRC population. Using bft as a marker, ETBF was detected more frequently in the stools of consecutive cases of CRC compared with that in concurrent hospital-based, age-, and gender-matched patients without CRC (38\% ETBF in 73 cases of CRC and $12 \%$ ETBF in 59 controls, $P<0.01)^{42}$. In the present study, we demonstrated a higher abundance of ETBF in CRC tissues compared with that in pairmatched normal tissues. Furthermore, we revealed high expression of BFAL1 in cancer tissues, which correlated positively with ETBF abundance. These findings indicated that direct exposure of colorectal epithelial cells (CECs) to ETBF may result in the upregulation of certain lncRNAs, including BFAL1 in CECs. In CRC cells, we observed that the BFAL1 mRNA level was upregulated by ETBF treatment. Therefore, we hypothesized that BFAL1 is responsible for ETBF stimulation of CRC and focused on BFAL1 for further study.

In a clinical study, we found that ETBF and BFAL1 have similar clinicopathological effects on tumor size. In addition, patients with high levels of ETBF and BFAL1 expression had a poor prognosis. These findings strongly suggested the emerging prognostic and therapeutic value of ETBF and BFAL1.

Initiation and progression of CRC refers to events yielding biological changes that foster CEC proliferation and multiple gene mutations or epigenetic alterations, ultimately resulting in the transformation to cancer. However, the events that precipitate in this process remain unknown. The microbiome is a prime suspect for triggering the initiation and/or progression of CRC carcinogenesis ${ }^{7}$. Recent studies have demonstrated that the gut microbiota exerts various biological functions on tumor formation and progression. Fusobacterium nucleatum was confirmed to induce autophagy and mediate 

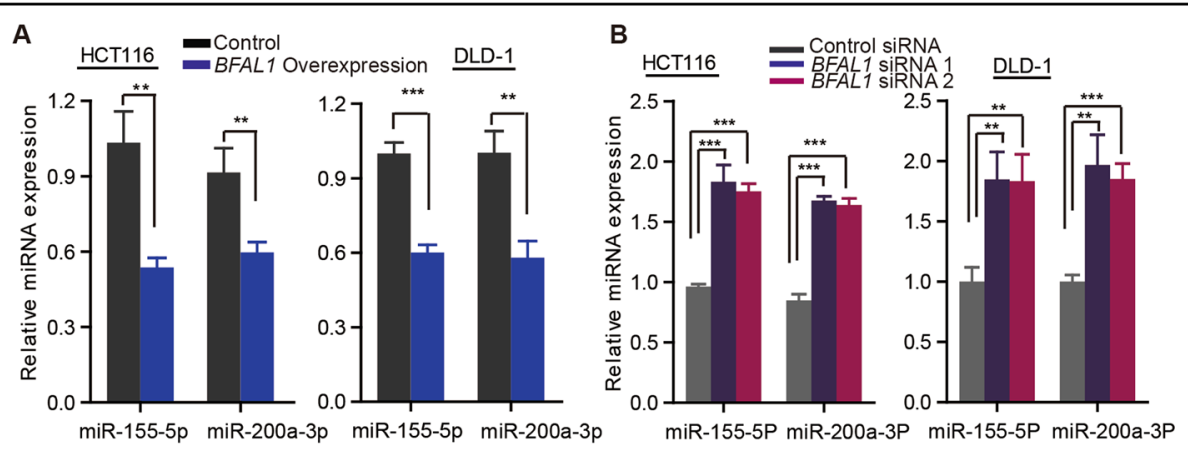

C

D
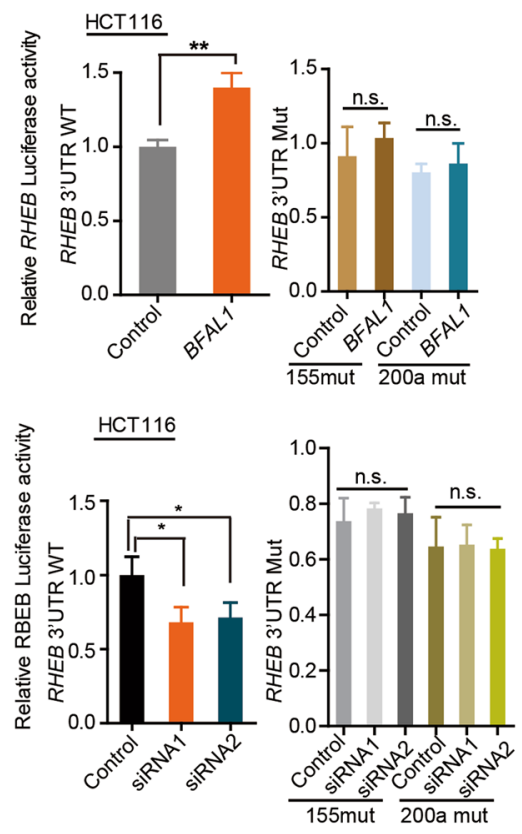

E

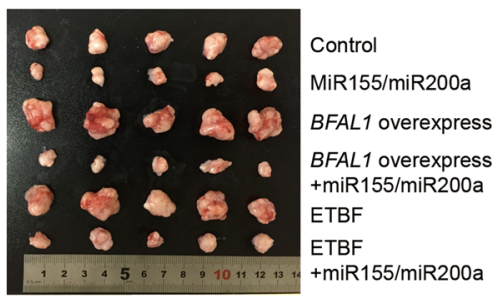

G

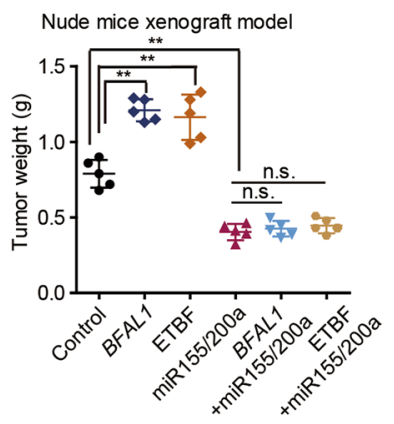

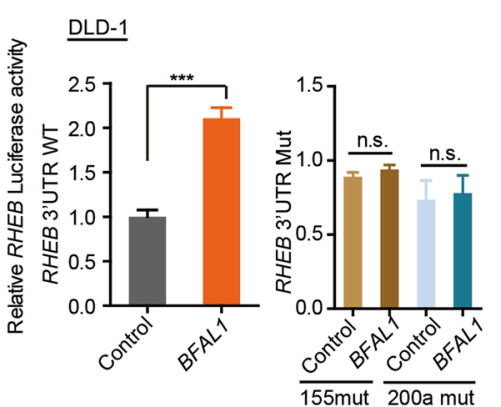
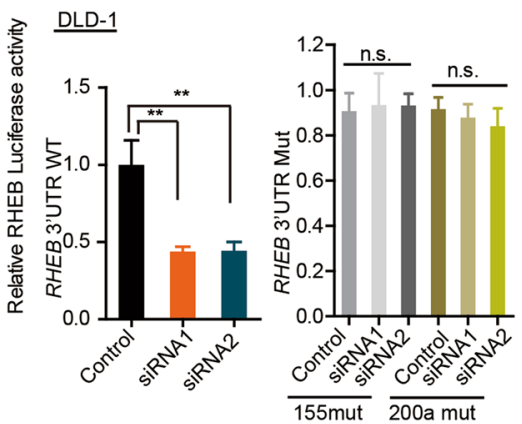

F

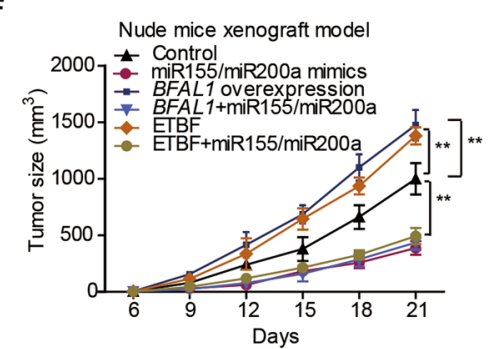

Fig. 5 (See legend on next page.) 
(see figure on previous page)

Fig. 5 BFAL1 regulates RHEB expression by sponging miR-155-5p and miR-200a-3p. a The miR-155-5p and miR-200a-3p mRNA levels in HCT116 cells and DLD-1 cells overexpressing BFAL1. $\mathbf{b}$ The miR-155-5p and miR-200a-3p mRNA levels in HCT116 cells and DLD-1 cells with BFAL1 knockdown. $\mathbf{c}$ Luciferase reporter assays were performed in HCT116 cells and DLD-1 cells overexpressing BFAL1. Luciferase reporters expressing wildtype or mutant human RHEB $3^{\prime}$ UTR were used (mean \pm SD of three independent experiments, ANOVA, ${ }^{*} P<0.05,{ }^{* *} P<0.01$, ${ }^{* * *} P<0.001$ ). d Luciferase reporter assays were performed in HCT116 cells and DLD-1 cells with BFAL1 knockdown. Luciferase reporters expressing wild-type or mutant human RHEB 3' UTR were used (mean \pm SD of three independent experiments, ANOVA, ${ }^{*} P<0.05,{ }^{* *} P<0.01,{ }^{* *} P<0.001$ ). e Xenograft tumors in the nude mouse model under different treatments $(n=5)$. f Statistical analysis of tumor sizes (mean $\pm S D, n=5$, ANOVA, ${ }^{*} P<0.01$ ). $g$ Analysis of tumor weights in different groups (mean $\pm \mathrm{SD}, n=5$, ANOVA, **P<0.01)

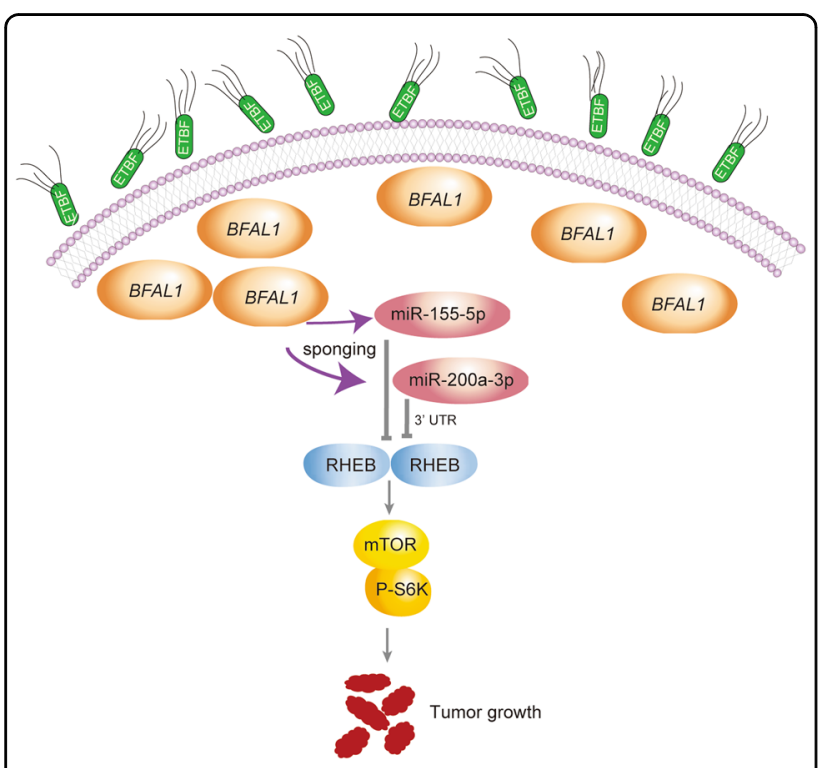

Fig. 6 Schematic diagram of ETBF-BFAL1 functions in CRC tumor growth. ETBF may stimulate BFAL1 overexpression, which competitively binds with miR-155-5p and miR-200a-3p, resulting in the activation of the RHEB/mTOR pathway, ultimately promoting CRC tumor growth

CRC chemoresistance ${ }^{27}$, and is also associated with metastasis of primary CRC ${ }^{43}$. The present study revealed that ETBF exerts a marked effect on CRC tumor growth in vitro and in vivo. However, when BFAL1 was knocked down, ETBF lost its biological effect on CRC, suggesting that BFAL1 mediates ETBF's carcinogenic function in CRC.

Further mechanistic investigations revealed a hitherto unidentified signaling cascade of ETBF-BFAL1 RHEB/ mTOR in CRC carcinogenesis. The mTOR-signaling pathway is reported to be dysregulated in $~ 50 \%$ of all human malignancies. This pathway acts as a sensor that integrates intracellular and extracellular events concerning metabolism and nutrients, and coordinates cell growth and autophagy ${ }^{44}$. RHEB is a regulator of mTOR and is indispensable for mTOR activation in response to all stimuli ${ }^{45}$. RHEB binds to mTOR on the lysosome surface and inactivates downstream signaling ${ }^{46}$. mTOR signaling is rarely reported in microbiota-associated CRC issues. Our findings revealed the exact mechanism of the ETBF-induced mTOR pathway mediated by BFAL1. BFAL1 regulates RHEB expression by binding competitively to miR-155-5p and miR-200a-3p, potentially acting as a ceRNA. Recent studies have provided evidence that gut microbiota might modulate miRNAs to exert biological functions. For example, F. nucleatum might modulate the expression of miR-18* and miR-4082 ${ }^{27}$, and Escherichia coli might modulate miR-30C and miR-130A to regulate human colonic epithelial cell autophagy ${ }^{47}$. By contrast, lncRNAs have been reported to bind with particular miRNAs to attenuate their suppressive effect on the target gene ${ }^{48,49}$. For example, precise regulation by IncRNA uc.173 was demonstrated in the control of gut permeability by decreasing the availability of miR-29b to regulate CLDN1 mRNA expression ${ }^{50}$. In the present study, we confirmed that BFAL1 regulates RHEB expression by binding to miR-155-5p and miR-200a-3p in ETBF-associated carcinogenesis.

Taken together, ETBF orchestrates the BFAL1, miR155-5p/miR-200a-3p, and the RHEB/mTOR pathways to regulate CRC tumor growth. LncRNA BFAL1 might be a promising target for CRC diagnosis and therapy.

\section{Acknowledgements}

This work was supported by the Division of Gastroenterology and Hepatology, Renji Hospital, School of Medicine, and Shanghai Jiao Tong University. This study was financially supported by the National Natural Science Foundation of China (Grant Nos. 81421001, 81530072, 81871901, 81874159, and 81790632).

\section{Author details}

${ }^{1}$ State Key Laboratory for Oncogenes and Related Genes; Key Laboratory of Gastroenterology \& Hepatology, Ministry of Health; Division of Gastroenterology and Hepatology; Shanghai Institute of Digestive Disease; Renji Hospital, School of Medicine, Shanghai Jiao Tong University, 145 Middle Shandong Road, 200001 Shanghai, China. ${ }^{2}$ Department of Infectious Disease, Shanghai Ninth People's Hospital, Shanghai Jiao Tong University School of Medicine, 639 Zhizhaoju Road, 200001 Shanghai, China. ${ }^{3}$ Department of Gastrointestinal Surgery, Renji Hospital, School of Medicine, Shanghai Jiao Tong University, 200001 Shanghai, China

\section{Conflict of interest}

The authors declare that they have no conflict of interest.

\section{Publisher's note}

Springer Nature remains neutral with regard to jurisdictional claims in published maps and institutional affiliations. 
Supplementary Information accompanies this paper at (https://doi.org/ 10.1038/s41419-019-1925-2).

Received: 4 February 2019 Revised: 2 August 2019 Accepted: 12 August 2019

Published online: 12 September 2019

\section{References}

1. Labianca, R. et al. Colon Cancer. Crit. Rev. Oncol/Hematol. 74, 106-133 (2010).

2. Smith, R. A., Cokkinides, V., Brooks, D., Saslow, D. \& Brawley, O. W. Cancer screening in the United States, 2010: a review of current American cancer society guidelines and issues in cancer screening. CA: A Cancer J. Cilni. 60 99-119 (2010).

3. Siegel, R., Desantis, C. \& Jemal, A. Colorectal cancer statistics, 2014. CA: A Cancer J. Clini. 64, 104-117 (2014)

4. Terzic, J., Grivennikov, S., Karin, E. \& Karin, M. Inflammation and colon cancer. Gastroenterology 138, 2101-2114 (2010).

5. Viaud, S. et al. The intestinal microbiota modulates the anticancer immune effects of cyclophosphamide. Science 342, 971-976 (2013).

6. Vetizou. et al. Anticancer immunotherapy by CTLA-4 blockade relies on the gut microbiota. Science 350, 1079-1084 (2015).

7. Sears, C. L. \& Garrett, W. S. Microbes, microbiota, and colon cancer. Cell Host Microbe 15, 317-328 (2014).

8. DeStefano Shields, C. E. et al. Reduction of murine colon tumorigenesis driven by Enterotoxigenic Bacteroides fragilis using cefoxitin treatment. J. Infect. Dis. 214, 122-129 (2016).

9. Peloquin, J. M. \& Nguyen, D. D. The microbiota and inflammatory bowel disease: insights from animal models. Anaerobe 24, 102-106 (2013).

10. Liam Chung. et al. Bacteroides fragilis toxin coordinates a procarcinogenic inflammatory cascade via targeting of colonic epithelial cells. Cell Host Microbe 23, 1-12 (2018).

11. Wu, S., Dreyfus, L. A., Tzianabos, A. O., Hayashi, C. \& Sears, C. L. Diversity of the metalloprotease toxin produced by Enterotoxigenic Bacteroides fragilis. Infect. Immun. 70, 2463-2471 (2002).

12. Sears, C. L., Geis, A. L. \& Housseau, F. Bacteroides fragilis subverts mucosal biology from symbiont to colon carcinogenesis. J. Clin. Investig. 124, 4166-4172 (2014)

13. Hyum, C. R., Do, Y. Y., Su, H. K., Young, J. K. \& Jung, M. K. Bacteroides fragilis enterotoxin upregulates intracellular adhesion molecule-1 in endothelial cells via an eldose reductase-, MAPK-, and NF-KB-Dependent pathway, leading to monocyte adhesion to endothelial cells. J. Immunol. 187, 1931-1941 (2011).

14. Kun, Z. et al. The Iver-enriched Inc-LFAR1 promotes liver fibrosis by activating TGFß and Notch pathways. Nat. Commun. 8, 144-159 (2011).

15. Francesco, P. M., Ivan, R. \& Maite, H. The multidimensional mechanisms of long noncoding RNA function. Genome Biol. 18, 206-218 (2017).

16. Prensner, J. R. \& Chinnaiyan, A. M. The emergence of IncRNAs in cancer biology. Cancer Discov. 1, 391-404 (2011).

17. Yang, F. et al. Long noncoding RNA high expression in hepatocellular carcinoma facilitates tumor growth through enhancer of zeste homolog 2 in humans. Hepatology 54, 1679-1689 (2011).

18. Kretz, M. et al. Control of somatic tissue differentiation by the long non-coding RNA TINCR. Nature 493, 231-235 (2013).

19. Yoon, J. H., Abdelmonhsen, K. \& Gorospe, M. Posttranscriptional gene regulation by long noncoding RNA. J. Mol. Biol. 425, 3723-3730 (2013).

20. Quinn, J. J. \& Chang, H. Y. Unique features of long non-coding RNA biogenesis and function. Nat. Rev. Genet. 17, 47-62 (2016).

21. Yufeng, Q. \& Paul, A. W. Crosstalk between the microbiome and epigenome messages from bugs. J. Biochem. 163, 105-112 (2018).

22. Nicholson, J. K. et al. Host-gut microbiota metabolic interactions. Science $\mathbf{3 3 6}$ 1262-1267 (2012).

23. Paul, B. et al. Influences of diet and the gut microbiome on epigenetic modulation in cancer and other diseases. Clin. Epigenetics 7, 112 (2015).

24. Liang, L. X., Ai, L. Y., Qian, J., Fang, J. Y. \& Xu, J. Long noncoding RNA expression profiles in gut tissues constitute molecular signatures that reflect the types of microbes. Sci. Rep. 5, 11763 (2015).
25. Pantosti, A., Melpeli, M., Wilks, M., Menozzi, M. J. \& D'Ambrosio Detection of enterotoxigenic Bacteroides fragilis by PCR. J. Clin. Microbiol. 35, 2482-2486 (1997).

26. Antunes, E. N. F. et al. Pattern III non-toxigenic Bacteroides fragilis (NTBF) strains in Brazil. Anaerobe 8, 17-22 (2002).

27. Xiaomeng, L., Yenshou, L., Sara, O. V., Kazuyoshi, Y. \& Joseph, A. Rheb binds and regulates the mTOR kinase. Curr. Biol. 15, 702-713 (2005).

28. Cesana, $\mathrm{M}$. et al. A long noncoding RNA controls muscle differentiation by functioning as a competing endogenous RNA. Cell 147, 358-369 (2011).

29. TaChung, Y. et al. Fusobacterium nucleatum promotes chemoresistance to colorectal cancer by modulating autophage. Cell 170, 548-563 (2017).

30. Lewis, B. P., Burge, C. B. \& Bartek, D. P. Conserved seed pairing, often flanked by adenosines, indicates that thousands of human genes are microRNA targets. Cell 120, 15-20 (2015).

31. Jan, K. \& Marc, R. RNAhybrid: microRNA target prediction easy, fast and flexible. Nucleic Acids Res. 34, 451-454 (2016).

32. $L V$, J. et al. Ox-LDL-induced microRNA-155 promotes autophagy in human endothelial cells via repressing the Rheb/mTOR pathway. Cell Physiol. Biochem. 42, 1436-1448 (2017)

33. Xing, G. et al. Influence of miR-155 on cell apoptosis in rats with ischemic stroke role of the ras homolog enriched in brain (Rheb)/mTOR pathway. Med Sci. Monit. 22, 5141-5153 (2016).

34. Caballero, G. E. et al. In vivo inhibition of miR-155 promotes recovery after experimental mouse stroke. J. Neurosci. 35, 12446-12464 (2015).

35. Wang, $Y$. et al. MicroRNA-199a-5p induced autophage and inhibites the pathogenesis of ankylosing spondylitis by modulating the mTOR signaling via directly targeting Ras Homolog Enriched in Brain (Rheb). Cell Physiol. Biochem. 42, 2481-2491 (2017)

36. Guo, W. et al. miR-200a regulates Rheb-mediated amelioration of insulin resistance after duodenal-jejunal bypass. Int J. Obes. (Lond.) 40, 1222-1232 (2016).

37. Qian, K. et al. Identifying autophage gene-associated module biomarkers through constrcution and analysis of an autophage- mediated ceRNAceRHA interaction network in colorectal cancer. Int J. Oncol. 53, 1083-1093 (2018).

38. Zhu, H., Zheng, T., Yu, J., Zhou, L. \& Wang, L. LncRNA XIST accelerates cervical cancer progression via upregulating fus through competitively binding with miR-200a. Biomed. Pharmacother. 14, 789-797 (2018).

39. Bultman, S. J. \& Jobin, C. Microbial-derived butyrate: an oncometabolite or tumor-suppressive metabolite? Cell Host Microbe 16, 143-145 (2014).

40. Wu, X. \& Zhang, Y. TET-mediated active DNA demethylation: mechanism, function and beyond. Nat. Rev. Cenet. 18, 517-534 (2017).

41. Feil, R. \& Frage, M. F. Epigenetics and the environment: emerging patterns and implications. Nat. Rev. Genet. 13, 97-109 (2012).

42. Toprak, N. U. et al. A possible role of Bacteroides fragilis enterotoxin in the aetiology of colorectal cancer. Clin. Microbiol. Infect. 12, 782-786 (2006).

43. Susan, B. et al. Analysis of Fusobacterium persistence and antibiotic response in colorectal cancer. Science 358, 1443-1448 (2017).

44. Zhang, Y. J., Duan, Y. \& Zheng, X. F. Targeting the mTOR kinase domain: the second generation of mTOR inhibitors. Drug Discov. Today 16, 7-8 (2011).

45. Wang, X. W. \& Zhang, Y. J. Targeting mTOR network in colorectal cancer therapy. World J. Gastroenterol. 20, 4178-4188 (2014).

46. Sang, G. K., Gwen, R. B. \& John, B. Nutrient regulation of the mTOR Complex 1 signaling pathway. Mol. Cells 35, 463-473 (2013).

47. Nguyen, H. T., Dalmasso, G., Muller, S. \& Darfeuille, M. Crohn's diseaseassociated adherent invasive Escherichia coli modulate levels of microRNAs in intestinal epithelial cells to reduce autophage. Gastroenterology 146, 508-519 (2014).

48. Quan, $H$. et al. LncRNA-AK13185 sponges MiR-93-5p in newborn and mature osteoclasts to enhance the secretion of vascular endothelial growth factor a promoting vasculogenesis of endothelial progenitor cells. Cell. Physiol. Biochem. 46, 401-417 (2018).

49. Yuan, J. H. et al. A long noncoding RNA activated by TGF- $\beta$ promotes the invasion-metastasis cascade in hepatocellular carcinoma. Cancer Cell 25, 1-16 (2014).

50. Wang, J. Y. et al. Regulation of intestinal barrier function by IncRNA Uc.173 through interaction with miR-29b. Mol. Cell. Biol. 38, 10-18 (2018). 